\title{
Editorial
}

\section{Advances in Electrospun Nanofibers}

\author{
Niranjan Patra, ${ }^{1}$ Miroslav Cernik, ${ }^{2}$ and Marco Salerno ${ }^{3}$ \\ ${ }^{1}$ Department of Materials, Imperial College London, London SW7 2AZ, UK \\ ${ }^{2}$ Centre for Nanomaterials, Technical University of Liberec, 46117 Liberec, Czech Republic \\ ${ }^{3}$ Nanophysics Department, Istituto Italiano di Tecnologia, 16163 Genova, Italy
}

Correspondence should be addressed to Niranjan Patra; n.patra@imperial.ac.uk

Received 1 August 2016; Accepted 1 August 2016

Copyright (C) 2016 Niranjan Patra et al. This is an open access article distributed under the Creative Commons Attribution License, which permits unrestricted use, distribution, and reproduction in any medium, provided the original work is properly cited.

With this special issue of $A E N F$ we are pleased to present contributions to the exciting field of electrospinning of nanofibers and its applications. Among different methods for producing one-dimensional (1D) nanostructures, electrospinning is the simplest, most economically viable, and commercially successful process of generating nanofibers and is continuously rising in research and developments. Rapid progress is being made especially in the area of electrospun nanofiber applications forming a bridge among materials science, biomedicine, and electronics. In this special issue, we have seven excellent contributions forming an interesting collection of works on nanofibers spinning and its disparate applications in biomedicine, decontamination process, and materials science.

A review article "Carbon Nanotube and Graphene Based Polyamide Electrospun Nanocomposites" comes from F. Navarro-Pardo et al., where the authors present a nice overview of the process of forming amide electrospun nanocomposites fibers using two well-known carbon nanostructures, namely, CNTs or graphene, as the fillers, which qualify for diverse field of applications like drug delivery system, biosensors, solar cells, electronic devices, transparent electrodes, or membrane filtration. This review elaborates the process parameters, especially how the selection of solvent is important for the dispersion of carbon nanostructures in the matrix to form nanofibers. The authors also discuss electrical, optical, and mechanical properties as well as crystallinity of the composite obtained by a number of research groups. The comprehensive picture given by F. Navarro-Pardo et al. may help to understand the structure-property relationship of polyamide/CNT/graphene based spun fiber composites from which in the future new applications would probably stem given their exceptional properties.

The use of $\mathrm{NaCl}$ solution as shell fluid to prepare medicated nanofibers in a modified coaxial electrospinning is presented by $\mathrm{Y}$. Wu et al. These authors have modified and developed a coaxial electrospinning to produce medicated nanofibers with the help of $\mathrm{NaCl}$ as shell fluid for ease of preparation. They found that the shell-to-core ratio of the fluid flow rate plays an important role in controlling the fiber diameter and morphology, as observed by optical and scanning electron microscopy. The fabricated nanofibers have a fine compatibility with sodium Diclofenac and can elute the drug in neutral condition, suggesting potential application in colon targeted drug release. $\mathrm{Y}$. Wu et al. have also carried out ex vivo tests to prove that the mats enhance the transmembrane delivered drug. Based on the above facts, the authors claim development of a potential medicated nanomaterial with tunable diameters and improved functional performance.

S. Seo et al. present an overview of the applications of spun nanofibers in dentistry. They have especially focused on polymeric nanofibers or bioceramic nanoparticle-incorporated nanofibers. The article shows how these advanced materials have contributed to highly promotive cell homing behavior and improved dental tissue regeneration. It also describes how the spun nanofibers play a versatile role in controlled release of biomolecule therapeutics (i.e., growth factors) or modification with adhesive biomolecules (i.e., fibronectin and RGD sequence) and contributes to further improved dental regeneration. Whereas a number of experiments on nanofibrous scaffolds in the in vitro and in vivo study 
have already been conducted, clinical customization to each patient's defect is still difficult. Additionally, since dental tissue degeneration may come from biological disorders, further studies of biological interplay between electrospun nanofiber and cells derived from compromised dental tissue are essential. Further studies will help to understand the biological effect of nanofibers, which can conclusively elaborate techniques to customize nanofiber scaffolds and categorize clinical defects into several groups for their customization. The work of S. Seo et al. sheds light on recently applied spun nanofibers in dentistry from process to applications.

The work of T. Jiříček et al. entitled "Flux Enhancement in Membrane Distillation Using Nanofiber Membranes” studied the membrane distillation. Spun PVDF nanofibers were tested under various conditions on a direct contact MD unit to find the optimum conditions for flux, which is compared with the commercially available membrane of PTFE, PE, and PES. Membrane thickness proved to be a crucial parameter when fluxes are concerned. They have found that the thinner membranes have higher fluxes and lower distillate purity. Also higher energy losses via conduction are found for thinner membranes. The authors suggest that since mass and heat transfers are connected, it is best to develop new membranes with a target application in mind for the specific membrane module and operational conditions. It is also shown that flux, energy efficiency, and distillate purity are closely connected, and one cannot be increased without sacrificing the other two. Therefore, nanofiber membranes suggest a solution but further improvement of membrane hydrophobicity in terms of LEP would be required for most future large scale applications.

On the other hand, A. Vojtěch et al. studied the organochlorinated pesticides decontamination using polyetherimide nanofibers as a sorbent, fixed on a solid phase microextraction (SPME) assembly made of steel wire, and compared with three commercial available SPME fibers, that is, PDMS $7 \mu \mathrm{m} / 100 \mu \mathrm{m}$ and DVB/Carboxene/PDMS, in which extraction time variability parameter was solely focused. Organochlorinated pesticides hexachlorocyclohexanes $(\mathrm{HCH})$ and chlorobenzenes $(\mathrm{ClB})$ were chosen as model water pollutants. The work presents the thermal, morphological, and adsorption properties of the nanofibers. In particular the authors observed that the polyetherimide (PEI) fibers show improved response for the target compound compared to other commercial available fibers, which allows shortening the extraction time from 50 to 10 mins, while maintaining the required sensitivity. This fast sorption and economical and easy production of PEI nanofibers would be most beneficial for using them as sorbent in the SPME fibers. The authors also suggest further research in polyetherimide nanofibers as a sorbent in analytical chemistry.

I. Veverkova and I. Lovětinská-Šlamborová present a study based on antibacterial activities using modified silica nanofibers. The authors have developed a functionalized organic-inorganic nanofibrous material which has biomedical application as wound dressing material for skin regeneration. Nanofibrous membrane of a combination of water-soluble polymer PVA with silica was electrospun and stabilized by heat-treatment before being functionalized with nanoparticles of silver and copper. Cut to the chase, the prepared functionalized nanofibrous membrane shows good antibacterial activities, having high potential as a wound dressing material favoring tissue regeneration.

I. Lovětinská-Šlamborová et al. developed a nontoxic silica nanofiber which is immobilized with topical antibiotic (tetracycline) in two different methods (spectrophotometric analysis and HPLC analysis) for quantification onto silica fibers. They have found that covalent functionalization works better than the simple physisorption in immobilization of tetracycline into the silica fibers. The tetracycline immobilized nanofibers membrane shows excellent antibacterial behavior which is intended for biomedical application. The tested quantity of antibiotics is much less than the commercial available tetracycline which ruled out the fear of antibiotics resistance to human body when used in higher quantity.

While the papers in this special issue illustrate some interesting applications which can open up new windows for the scientific community and for the betterment of the mankind, there is still much room for further development and improvement of the properties of these materials, to maximize their utility and spread their use further. This may be regarded as a small step towards a much bigger and systematic investigation of electrospun nanofibrous materials for new applications.

\section{Acknowledgments}

We are grateful to the authors who contributed their works for this special issue and worked proactively with us during manuscript revisions. We are also thankful to all reviewers for their work on the manuscripts.

Niranjan Patra Miroslav Cernik Marco Salerno 

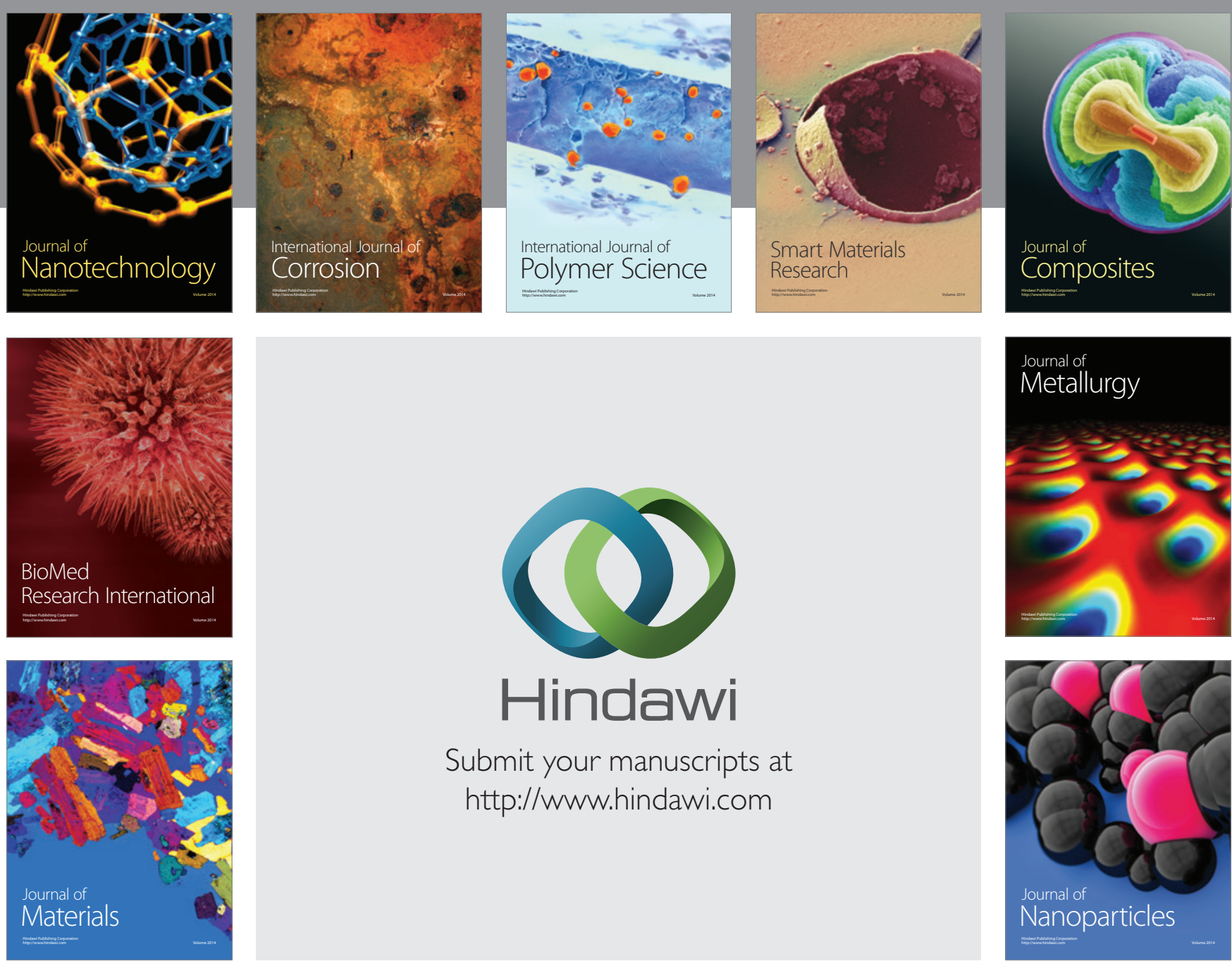

\section{Hindawi}

Submit your manuscripts at

http://www.hindawi.com

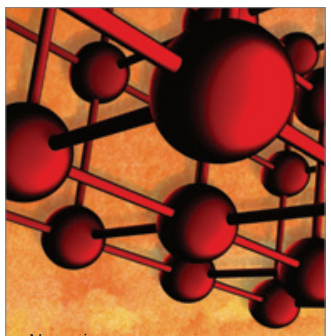

Materials Science and Engineering
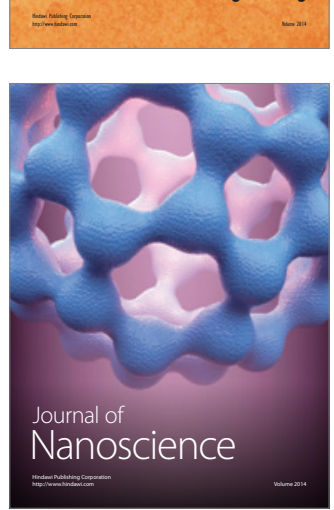
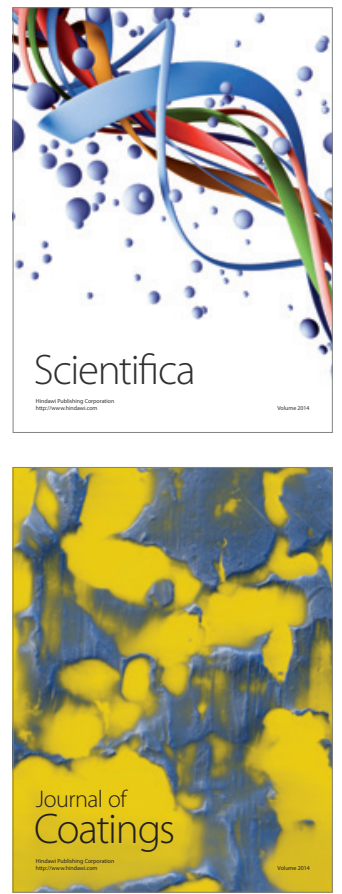
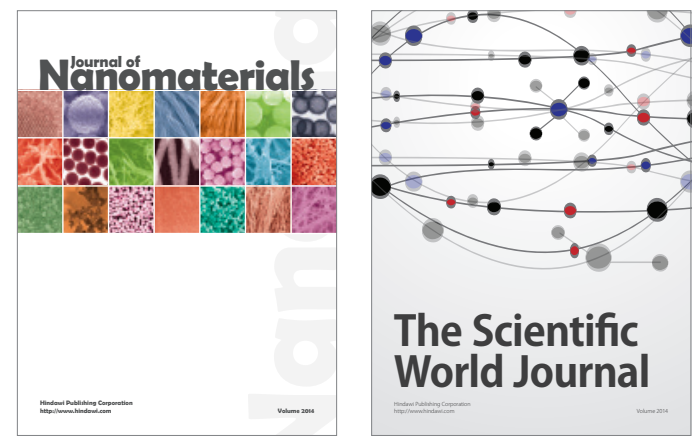

The Scientific World Journal
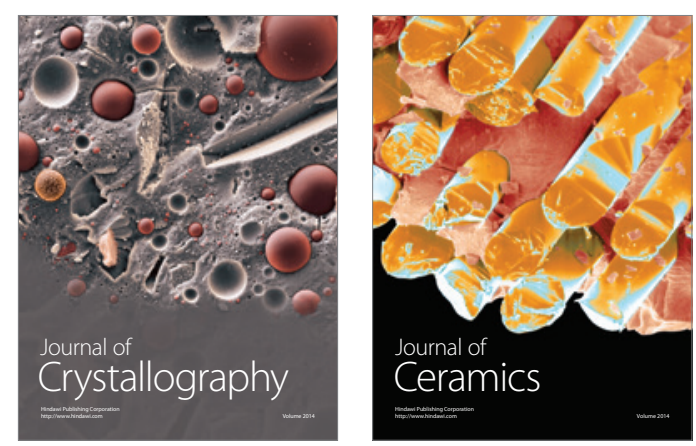
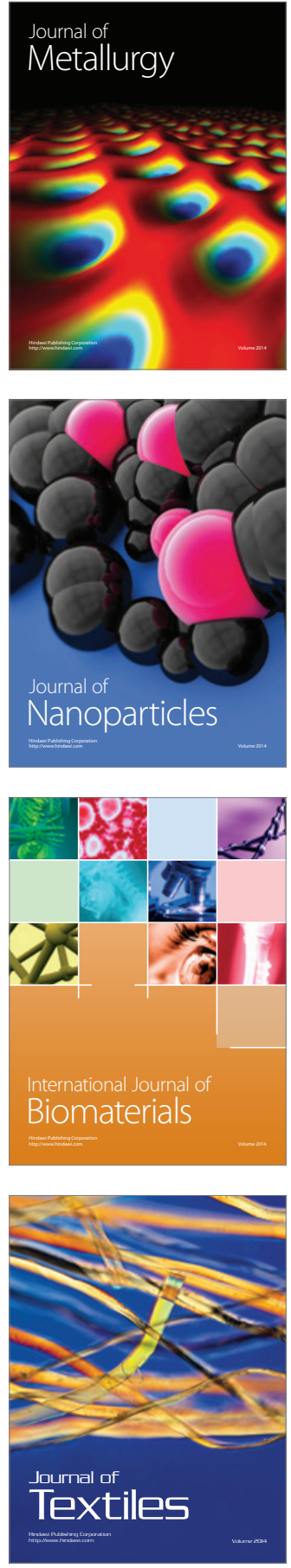